\title{
Montelukast in Allergic Rhinitis: A Review
}

\author{
Chowdhury MAN ${ }^{1}$, Uddin $\mathrm{MS}^{2}$, Das $\mathrm{S}^{3}$, Hoque $\mathrm{M}^{4}$
}

\begin{abstract}
Leukotriene inhibitors are the first new class of medications for the treatment of persistent asthma that have been approved by the U.S food and drug Administration in more than two decades. They also have been approved for the treatment of Allergic Rhinitis. Leukotrienes are inflammatory mediators that are known as the slow-reacting substance of anaphylaxis. Synthesis of these mediators results from the cleavage of arachidonic acid in cell membranes, exert their biologic effect by binding and activating specific adaptors. This occurs in a series of events that lead to their important role in the Allergic Rhinitis. Because agents lead to the production of symptoms, the use of leukotriene inhibitors, particularly montelukast seems appropriate. These classes of drug can block the binding of leukotrienes to CysLT1 receptors and can also result in the prevention of leukotriene activity. Montelukast is the most commonly used inhibitor of the leukotriene pathway because of its ease of use, good safety profile, and once-daily regimen.
\end{abstract}

We conduct a systematic review of studies that have evaluated montelikast in the treatment of Allergic Rhinitis. This review focuses on the pathophysiology and effect of montelukast in Allergic Rhinitis. The purpose of this paper is to provide an overview of the accumulation data concerning these agents in treating Allergic Rhinitis. Montelukast provides an effective and well tolerated oral treatment for Allergic Rhinitis.

\section{Introduction}

Allergic rhinitis and allied condition are common disease which require active therapy, as it is a nuisance to have a constant nasal irritation, excessive discharge and blockage

1. Corresponding Author: Dr. Md Abu Nayeem Chowdhury Associate Professor of Pharmacology Sylhet Women's Medical College, Sylhet

2. Corresponding Author: Dr. Md Samir Uddin Assistant Professor of Anatomy Sylhet Women's Medical College, Sylhet

3. Dr. Suprava Das Assistant Professor of Pharmacology Sylhet Women's Medical College, Sylhet

4. Dr. Mozzamel Hoque Associate Professor of Otolaryngology Sylhet Women's Medical College, Sylhet with nasal voice which is unacceptable to for example a school teacher or a telephone operator ${ }^{1}$. Seasonal allergic rhinitis or hay fever was called "catarrhus aestivus" (spring catarrh) in 19th century medical literature, but more recently has been labelled as a "post industrial revolution epidemic" ". Its prevalence has increased in developed countries, particularly in the past two decades ${ }^{2}$. One in six people is affected by allergic rhinitis ${ }^{2}$. Allergic rhinitis is an inflammatory disorder associated with local leukotriene release during periods of symptoms ${ }^{3}$. Leukotrienes are inflammatory mediators that are known as the slow-reacting substance of anaphylaxis produced by a number of cell types including mast cells, eosinophils, basophils, macrophages, and monocytes. Synthesis of these mediators results from the cleavage of arachidonic acid in cell membranes, and they exert their biologic effects by binding and activating specific adaptors. This occurs in a series of events that lead to contraction of the human airway smooth muscle, chemotaxis, and increased vascular permeability. These effects have led to their important role in the diseases of asthma, allergic rhinitis. Leukotrienes have been known in the field of immunology since the 1930s. At that time they were referred to as the slow-reacting substance of anaphylaxis. They were not, however, characterized until the 1980s, where they were noted to be formed during the breakdown of arachidonic acid by the enzyme 5lipoxygenase. They consist of LTA ${ }_{4}, \mathrm{LTB}_{4}, \mathrm{LTC}_{4}, \mathrm{LTD}_{4}$, and $\mathrm{LTE}_{4}$, and they were named because the apparent molecule was originally isolated from leukocytes and therefore its carbon backbone contains three double bonds in series, which constitutes a trion. This structural information provided the key to the oxidative pathway of lipometabolism, known as the 5-lipoxygenase. They are classified as inflammatory mediators, and are produced by a number of cell types, particularly mast cells, eosinophils, basophils, macrophages, and monocytes. With the identification of asthma, allergic rhinitis, and paranasal sinusitis associated with the inflammatory pathways, the leukotrienes have been implicated in the pathogenesis of these conditions and have become targets for therapeutic modulation ${ }^{4}$. Cysteinyl-leukotrienes (CysLTs) are endogenous mediators of inflammation and play an important role in allergic airway disease by stimulating bronchoconstriction, mucus production, mucosal oedema and inflammation, airway infiltration by eosinophils, and dendritic cell maturation that prepares for future allergic response. Montelukast inhibits these actions by blocking type $^{1}$ CysLT receptors found on immunocytes, smooth muscle and endothelium in the respiratory mucosa ${ }^{5}$. 


\section{Risk factors}

Allergic rhinitis is a type I immediate hypersensitivity reaction mediated by specific IgE antibody to a seasonal allergen, leading to mucosal inflammation characterised by sneezing, itching, rhinorrhoea, and nasal blockage. Pollens from wind pollinated grasses, trees, weeds, and spores from fungi are the commonest aeroallergens. Pollens are the male gametes of plants, and most antigenic pollens are 6-40 $\mu \mathrm{m}$ in diameter. Larger pollens from oil seed rape, which is pollinated by insects, have recently been implicated as causative agents ${ }^{6}$. Grass pollen is the commonest cause of seasonal allergic rhinitis in Britain. The highest levels of pollen in the atmosphere are found in May, June, and July. Flowering of plants occurs once every 4-12 days, mostly in the morning ( 4 am to 9 am). Pollen grains are carried high into the air around the middle of the day and fall in the evening as the air cools. Pollen concentrations of 50 grains $/ \mathrm{mm}^{3}$ are associated with symptoms in all susceptible people. Fungal spores are more prevalent from July to September. Warm, dry, and clear conditions increase levels of atmospheric pollen and spores ${ }^{7}$.

\section{Prevalence}

The prevalence of seasonal allergic rhinitis is rising as all atopic diseases are increasing ${ }^{8}$. The peak months for seasonal allergic rhinitis are May, June, and July. A survey of 5349 adults in southwest London has shown the prevalence of seasonal symptoms to be $11 \%{ }^{9}$. Seasonal allergic rhinitis has a peak prevalence in adolescence and early adulthood ${ }^{8}$. There is no difference between the sexes in prevalence ${ }^{10}$. In Britain general practice consultations for hay fever have also risen ${ }^{11}$. The prevalence has regional variation in the frequency of the atopic genotype or an exposure to aeroallergens in early life. The prevalence is higher among children from affluent households ${ }^{12}$. In Japan a direct relation was found between sensitivity to pollen and proximity to areas with high levels of diesel exhaust particles in ambient air ${ }^{13}$. Animal studies suggest that these particles increase allergic sensitisation ${ }^{14}$.

\section{Clinical Diagnosis}

Diagnosis is made on a good clinical history. The cardinal symptoms of seasonal allergic rhinitis are sneezing, rhinorrhoea, itching, and nasal obstruction. Nasal discharge is clear and watery. Pruritus affects the nose, eyes, and palate. Nasal obstruction is more pronounced in the late phase reaction. Pollen contact with eyes causes soreness, and sensitive subjects show congestion and periorbital oedema. Some $13-38 \%$ of patients also have asthma ${ }^{8}$ and eczema $^{15}$.

\section{Pathophysiology}

On exposure to the allergen, antigenic proteins are dissolved in mucus and enter the nasal mucosa. They are engulfed by cells that present them to other immunocompetent cells ( $\mathrm{T}$ and B lymphocytes), with subsequent production of specific antibody. In atopic people the local cytokines (interleukin 4, interleukin 13) and T2 lymphocytes encourage an IgE response. The Fc portion of this IgE antibody binds to high affinity receptors on the surface of mast cells. Subsequent exposure to the allergen causes a cross linking of two or more IgE molecules and degranulation of mast cells. This leads to release of biochemical mediators which act on local cells, nerve endings, and vasculature ${ }^{16}$. Increased levels of proinflammatory mediators like histamine, tryptase, prostaglandin D2, and leukotrienes are seen in nasal lavage fluid after an allergen challenge. These mediators are derived from mast cells in the early phase. Plasma exudation is also a major feature in early phase reaction. This provides kinins, which play a role in pathogenesis. Eosinophil cationic protein, cytokines, and major basic protein are the predominant mediators in the late phase. Eosinophils seem to be responsible for ongoing inflammation ${ }^{17}$.

\section{Prevention}

Avoidance of the offending antigen is the primary way to prevent allergic rhinitis. Identify the responsible allergen(s) can help the individual to establish ways to avoid the specific allergen, whether it is an environmental allergen or a household allergen such as dust mite or pet dander. In addition, contact reactions caused by medications or cosmetics are treated best by avoidance. Therefore patients should be encouraged to identify the allergen that is causing their problem and to avoid it. Numerous treatment options have become available for the relief of acute symptoms. Avoidance should always be the first line in therapy but in most cases, is not practical, especially with pollen allergies $^{18}$.

\section{Pharmacological Management}

Treatment can be immunological (allergen avoidance, immunotherapy-formerly called Hypo sensitization), medical and surgical. The best treatment is therefore given by a physician with detailed knowledge of the allergic, pharmacological and morphological aspects of rhinitis ${ }^{1}$.

\section{Allergic rhinitis can be treated by}

- Topical antihistamines-Act by competitive inhibition of histamine at the $\mathrm{H} 1$ receptor and block effects of endogenously released histamine.

- Mast cell stabilizers-Inhibit sensitized mast cell degranulation when exposed to specific antigens by inhibiting the release of mediators from the mast cells and block calcium ions from entering the mast cell.

- Corticosteroids-Have both anti-inflammatory (glucocorticoid) and salt retaining (mineralocorticoid) properties. Glucocorticoids have profound and varied metabolic effects. In addition, these agents modify the body's immune response to diverse stimuli.

- Nonsteroidal anti-inflammatory drugs (NSAIDs)Their mechanism of action is believed to be through inhibition of the cyclooxygenase enzyme that is essential for the biosynthesis of prostaglandins, which results in vasoconstriction, decrease in vascular permeability and leukocytosis, and decrease intraocular pressure ${ }^{19}$. 
- Leukotriene inhibitors (Montelukast)-is the most commonly used inhibitor of the leukotriene pathway ${ }^{20}$.

\section{Antileukotrienes}

These fall into two groups-leukotriene receptor antagonists and inhibitors of leukotriene synthesis. Both are oral drugs and effective against rhinitis in clinical trials, with an efficacy similar to that of antihistamines ${ }^{16}$. Leukotrienes have been known in the field of immunology since the 1930s. They were not, however, characterized until the 1980s, where they were noted to be formed during the breakdown of arachidonic acid by the enzyme 5lipoxygenase. They consist of $\mathrm{LTA}_{4}, \mathrm{LTB}_{4}, \mathrm{LTC}_{4}, \mathrm{LTD}_{4}$, and $\mathrm{LTE}_{4}$, and they were named because the apparent molecule was originally isolated from leukocytes. Leukotrienes are inflammatory mediators that are known as the slow-reacting substance of anaphylaxis produced by a number of cell types including mast cells, eosinophils, basophils, macrophages, and monocytes. Synthesis of these mediators results from the cleavage of arachidonic acid in cell membranes, and they exert their biologic effects by binding and activating specific adaptors. This occurs in a series of events that lead to contraction of the human airway smooth muscle, chemotaxis, and increased vascular permeability. These effects have led to their important role in the diseases of asthma, allergic rhinitis, and possible paranasal sinusitis with the formation of nasal polyps. Because these agents lead to the production of symptoms in patients that are asthmatic, the use of leukotriene enzyme inhibitors, particularly montelukast seem appropriate. This drugs can block the binding of leukotrienes to CysLT1 receptors $^{21}$. Montelukast is the most commonly used inhibitor of the leukotriene pathway because of its ease of use, good safety profile, and once-daily regimen ${ }^{20,22}$.

Montelukast sodium is described chemically as:

[R-(E)]-1 - [ [ [ 1 - [3 - [2-( 7 - chloro-2quinolinyl) e the ny 1] phe ny 1]-3-[2-(1-hydroxy 1methylethyl)phenyl]propyl]thio]methyl]cyclopropaneaceti c acid, monosodium salt. The empirical formula is $\mathrm{C} 35 \mathrm{H} 35 \mathrm{ClNNaO} 3 \mathrm{~S}$, and its molecular weight is 608 .

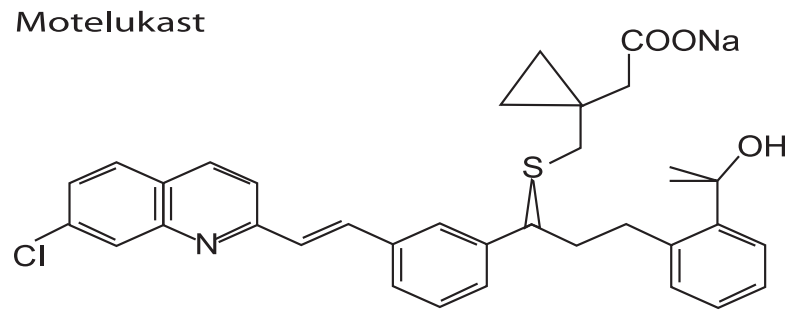

The structural formula is:

Montelukast sodium is a hygroscopic, optically active, white to off-white powder. Montelukast sodium is freely soluble in ethanol, methanol, and water ${ }^{13}$.

\section{Mechanism of Action}

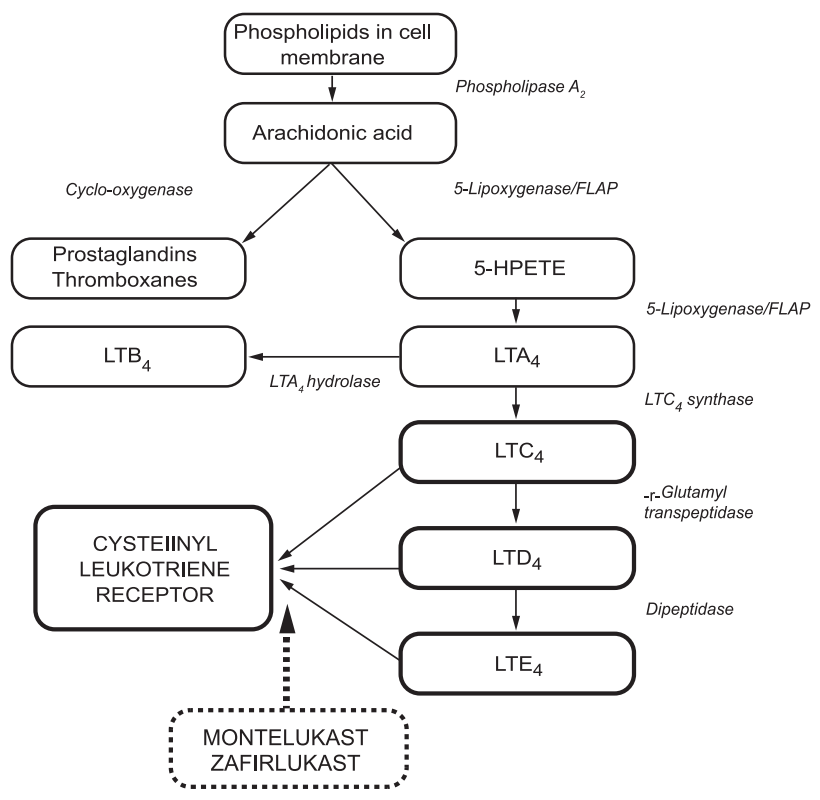

The cysteinyl leukotrienes $\left(\mathrm{LTC}_{4}, \mathrm{LTD}_{4}, \mathrm{LTE}_{4}\right)$ are products of arachidonic acid metabolism and are released from various cells, including mast cells and eosinophils. These eicosanoids bind to cysteinyl leukotriene (CysLT) receptors. The CysLT type-1 $\left(\mathrm{CysLT}_{1}\right)$ receptor is found in the human airway (including airway smooth muscle cells and airway macrophages) and on other pro-inflammatory cells (including eosinophils and certain myeloid stem cells). CysLTs have been correlated with the pathophysiology of asthma and allergic rhinitis. In asthma, leukotriene-mediated effects include airway edema, smooth muscle contraction, and altered cellular activity associated with the inflammatory process. In allergic rhinitis, CysLTs are released from the nasal mucosa after allergen exposure during both early-and late-phase reactions and are associated with symptoms of allergic rhinitis. Intranasal challenge with CysLTs has been shown to increase nasal airway resistance and symptoms of nasal obstruction. Montelukast is an orally active compound that binds with high affinity and selectivity to the $\mathrm{CysLT}_{1}$ receptor (in preference to other pharmacologically important airway receptors, such as the prostanoid, cholinergic, or $\beta$ adrenergic receptor). Montelukast inhibits physiologic actions of $\mathrm{LTD}_{4}$ at the CysLT 1 receptor without any agonist activity $^{23}$. Leukotriene inhibitors (Montelukast) have been approved by the U.S. Food and Drug Administration for the treatment of allergic rhinitis. Leukotriene inhibitors have minimal side effects and are well tolerated in most populations ${ }^{24}$. The use of montelukast in combination with antihistamines such as loratadine or cetirizine has generally resulted in greater efficacy than when these agents were used alone, and in some studies has produced results comparable with intranasally applied corticosteroids ${ }^{25}$. Studies show improvement in sneezing, nasal itching, or drainage during treatment with leukotriene receptor antagonists(Montelukast) ${ }^{26}$. 
The increasing prevalence of allergic rhinitis, its impact on individual quality of life and social costs, as well as its role as a risk factor for asthma, underline the need for improved treatment options for this disorder. We reviewed the recent literature published in English, dealing with relevant and appropriately controlled clinical trials on the treatment of allergic rhinitis. In addition to well established pharmacological therapies (with antihistamines, corticosteroids, decongestants and mast cell stabilizers), new therapeutic options with montelucast become increasingly important but further studies are still necessary.

\section{Reference}

1. Niels Mygind. Topical steroid treatment for allergic rhinitis and allied conditions. Clin. Otolaryngology.182;7:343-352.

2. McMenamin P. Costs of hay fever in the United States in 1990. Ann Allergy 1994;73:35-9.

3. TEET PULLERITS, LEA PRAKS, BENGT-ERIC SKOOGH, RAIVO ANI, and JAN LÖTVALL. Randomized Placebocontrolled Study Comparing a Leukotriene Receptor Antagonist and a Nasal Glucocorticoid in Seasonal Allergic Rhinitis. Am. J. Respir. Crit. Care Med. 1999;159:1814-1818.

4. Parnes, Steven M. MD. The role of leukotriene inhibitors in patients with paranasal sinus disease. Current Opinion in Otolaryngology \& Head \& Neck Surgery. 2003;11:184-191.

5. Nayak, Anjuli, Langdon, Ronald B. Montelukast in the Treatment of Allergic Rhinitis: An Evidence-Based Review. Source: Drugs. 2007;67:887-901.

6. McSharry C. Oilseed rape sensitivity. Clin Exp Allergy 1997;27:125-7.

7. Abhi Parikh, Glenis K Scadding. Clinical Review: Seasonal Allergic Rhinitis. BMJ 1997;314:1392

8. Aberg N. Asthma and allergic rhinitis in Swedish conscripts. Clin Exp Allergy 1989;19:59-63.

9. Sibbald B, Strachan D. Epidemiology of rhinitis. Blackwell Scientific, 1995:32-43.

10. Fleming DM, Crombie D. Prevalence of asthma and hay fever in England and Wales. BMJ 1987;294:279-83.

11. Ninan TK, Russell G. Respiratory symptoms and atopy in Aberdeen school children. BMJ 1992;304:873-5.

12. Romagnani S. Human TH1 and TH2 subsets: regulation of differentiation and role in protection and immunopathology. Int Arch Allergy Immunol 1992;98:279-85.
13. Ishizaka I, Koizumi K, Ikemori R, Ishiyama Y, Kushibiki E. Studies of prevalence of Japanese cedar pollinosis among the residents in a densely cultivated area. Ann Allergy 1987;58:265-70.

14. Takafuji S, Suzuki S, Muranaka M, Miyamoto T. Influence of environmental factors on IgE production. In: Chadwick D, Whelan J, eds. IgE, mast cells and allergic response. Chichester: John Wiley and Sons, 1989:199-203.

15. Gerrard J, Vickers P, Gerrard C. The familial incidence of allergic disease. Ann Allergy 1976;36:10-5.

16. Bousquet J, Vignola AM, Campbell AM, Michel FB. Pathophysiology of allergic rhinitis. Int Arch Allergy Immunol 1996;110:207-18.

17. Abhi Parikh, Glenis K Scadding. Clinical Review: Seasonal Allergic Rhinitis. BMJ 1997;314:1392.

18. Joss JD, Craig TJ. 'Seasonal allergic conjunctivitis: overview and treatment update', Journal of the American Osteopathic Association, 1999;99:13-13.

19. Majmudar PA. 'Conjunctivitis, Allergic', [Online], eMedicine World Medical Library, (Last update November 4, 2005). Available at http://www.emedicine.com/oph/topic85.htm [16 April 2007].

20. Scadding GK. Rhinitis Medicamentosa [Editorial]. Clin Exp Allergy 1995;25:391-4.

21. Parnes, Steven M. MD. The role of leukotriene inhibitors in patients with paranasal sinus disease. Current Opinion in Otolaryngology \& Head \& Neck Surgery. 2003;11:184-191.

22. Marc Peters-Golden, M.D., and William R. Henderson, Jr. M.D. Mechanisms of Disease, Leukotrienes. N Engl J Med. 2007;357:1841-1854.

23. O J Dempsey. Review of leukotriene receptor antagonist therapy. Postgrad Med J 2000;76:767-773.

24. Scow DT, Luttermoser GK, Dickerson KS. Leukotriene inhibitors in the treatment of allergy and asthma. American Family Physician. 2007;75:65-70.

25. Nayak, Anjuli; Langdon, Ronald B. Montelukast in the Treatment of Allergic Rhinitis: An Evidence-Based Review. Drugs, 2007;67:887-901

26. Robert A., Nathan MD. Pharmacotherapy for allergic rhinitis: a critical review of leukotriene receptor antagonists compared with other treatments. Annals of Allergy, Asthma \& Immunology. 2003;902:182-191. 